\title{
AN EXAMPLE OF AN ORTHOGONAL TRANSFORMATION OF HILBERT SEQUENCE SPACE
}

\author{
R. C. T. SMITH \\ (Received 10 November 1969) \\ Communicated by B. Mond
}

We use the equivalence of the sequence space $l_{2}$ and the function space $L_{2}$ to obtain an orthogonal transformation related to two series inversion formulae due, respectively, to Titchmarsh [1,2] and to Linfoot and Shepherd [3].

One interpretation of an orthogonal transformation in Euclidean space is that it gives the coordinates of a fixed point with respect to a new set of axes in terms of its coordinates with respect to the original axes. By analogy we consider the coordinates (Fourier coefficients) of an arbitrary function for two sets of orthogonal functions.

Let $\left\{\phi_{n}(x)\right\}$ be a complete set of orthogonal functions with weight function $w(x)$ so that

$$
\int_{a}^{b} w(x) \overline{\phi_{m}(x)} \phi_{n}(x) d x= \begin{cases}0, & m \neq n \\ 1, & m \neq n .\end{cases}
$$

Let $\left(a_{0}, a_{1}, a_{2}, \cdots\right)$ be a point in Hilbert sequence space. Then if $f(x) \sim \sum_{n=0}^{\infty}$ $a_{n} \phi_{n}(x)$ we have

$$
\sum_{n=0}^{\infty}\left|a_{n}\right|^{2}=\int_{a}^{b} w(x)|f(x)|^{2} d x .
$$

Again if $\left\{\Phi_{m}(x)\right\}$ is a second complete orthonormal system with weight function $W(x)$ over $(a, b)$, then if $g(x) \sim \sum_{m=0}^{\infty} b_{m} \Phi_{m}(x), \sum_{m=0}^{\infty}\left|b_{m}\right|^{2}<\infty$, we have

$$
\sum_{m=0}^{\infty}\left|b_{m}\right|^{2}=\int_{a}^{b} W(x)|g(x)|^{2} d x .
$$

If $f, g$ are such that $w^{\frac{1}{2}}(x) f(x)=W^{\frac{1}{2}}(x) g(x)$ a.e. in $(a, b)$, then

Moreover

$$
\sum_{n=0}^{\infty}\left|a_{n}\right|^{2}=\sum_{m=0}^{\infty}\left|b_{m}\right|^{2}
$$

$$
\begin{aligned}
b_{m} & =\int_{a}^{b} W(x) g(x) \overline{\Phi_{m}(x)} d x \\
& =\int_{a}^{b} W^{\frac{1}{2}}(x)\left\{w^{\frac{1}{2}}(x) f(x)\right\} \overline{\Phi_{m}(x)} d x \\
& =\sum_{n=0}^{\infty} a_{n} \int_{a}^{b} w^{\frac{1}{2}}(x) W^{\frac{1}{2}}(x) \phi_{n}(x) \overline{\Phi_{m}(x)} d x
\end{aligned}
$$


i.e.

$$
b_{m}=\sum_{n=0}^{\infty} k_{m n} a_{n}
$$

where

$$
k_{m n}=\int_{a}^{b} w^{\frac{1}{2}}(x) W^{\frac{1}{2}}(x) \overline{\Phi_{m}(x)} \phi_{n}(x) d x .
$$

Similarly

$$
a_{n}=\sum_{m=0}^{\infty} \overline{k_{m n}} \boldsymbol{b}_{m} .
$$

The method given above will generate interesting examples of unitary (or, in the real case, orthogonal) transformations only in exceptional cases. We now obtain a two parameter family of orthogonal transformations by use of suitable sets of Jacobi polynomials. Apart from normalizing factors these polynomials are completely determined by the conditions that $P_{n}^{(\alpha, \beta)}(x)$ is of degree $n$ for $n=0,1,2, \cdots$ and that

$$
\int_{-1}^{1}(1-x)^{\alpha}(1+x)^{\beta} P_{n}^{(\alpha, \beta)}(x) P_{m}^{(\alpha, \beta)}(x) d x=0
$$

for $m \neq n$, where $\alpha>-1, \beta>-1$. We use the same notation as [4].

$$
P_{n}^{(\alpha, \beta)}(x)=\left(\begin{array}{c}
n+\alpha \\
n
\end{array}\right) F\left(-n, n+\alpha+\beta+1 ; \alpha+1 ; \frac{1-x}{2}\right) .
$$

Then

$$
h_{n}(\alpha, \beta)=\int_{-1}^{1}(1-x)^{\alpha}(1+x)^{\beta}\left\{P_{n}^{(\alpha, \beta)}(x)\right\}^{2} d x=\frac{2^{\alpha+\beta+1} \Gamma(\alpha+n+1) \Gamma(\beta+n+1)}{n !(\alpha+\beta+2 n+1) \Gamma(\alpha+\beta+n+1)}
$$

and, in particular, for $n=0$

$$
h_{0}(\alpha, \beta)=\frac{2^{\alpha+\beta+1} \Gamma(\alpha+1) \Gamma(\beta+1)}{\Gamma(\alpha+\beta+2)} .
$$

Now let

$$
\begin{aligned}
& w(x)=(1-x)^{\alpha}(1+x)^{\beta}, \alpha>-1,0<\beta<1, \phi_{n}(x)=\frac{1}{\left\{h_{n}(\alpha, \beta)\right\}^{\frac{1}{2}}} P_{n}^{(\alpha, \beta)}(x) ; \\
& W(x)=(1-x)^{\alpha}(1+x)^{-\beta}, \quad \Phi_{m}(x)=\frac{1}{\left\{h_{m}(\alpha,-\beta)\right\}^{\frac{1}{2}}} P_{m}^{(\alpha,-\beta)}(x) .
\end{aligned}
$$

Then

$$
\begin{aligned}
k_{m n} & =\int_{-1}^{1} w^{\frac{1}{2}}(x) W^{\frac{1}{2}}(x) \phi_{n}(x) \Phi_{m}(x) d x \\
& =\frac{1}{\left\{h_{n}(\alpha, \beta) h_{m}(\alpha,-\beta)\right\}^{\frac{1}{2}}} \int_{-1}^{1}(1-x)^{\alpha} P_{n}^{(\alpha, \beta)}(x) P_{m}^{(\alpha,-\beta)}(x) d x \\
& =\frac{1}{\left\{h_{n}(\alpha, \beta) h_{m}(\alpha,-\beta)\right\}^{\frac{1}{2}}} \frac{(-1)^{m+n} \sin \pi \beta 2^{\alpha+1} \Gamma(\beta+n+1) \Gamma(-\beta+m+1)}{\pi m ! n !(\alpha+m+n+1)(\beta-m+n)}
\end{aligned}
$$


[This integral is a special case of

$$
\begin{aligned}
\int_{-1}^{1}(1-x)^{\alpha}(1+x)^{\beta+\sigma} P_{n}^{(\alpha, \beta)}(x) P_{m}^{(\alpha, \sigma)}(x) d x \\
\quad=\frac{2^{\alpha+\beta+\sigma+1} \Gamma(\alpha+m+n+1) \Gamma(\beta+n+1) \Gamma(\beta+\sigma+1) \Gamma(\sigma+m+1)}{m ! n ! \Gamma(\alpha+\beta+\sigma+m+n+2) \Gamma(\beta-m+n+1) \Gamma(\sigma+m-n+1)},
\end{aligned}
$$

formula (13), p. 286 in [4].]

Finally we write

$$
\begin{aligned}
& (-1)^{n} a_{n}=a_{n}^{*},(-1)^{m} b_{m}=b_{m}^{*}, \\
& k_{m n}=\frac{(-1)^{m+n} 2^{\alpha+1}}{A_{n} B_{m}} \frac{\sin \pi \beta}{\pi} \frac{1}{(\alpha+m+n+1)(\beta-m+n)},
\end{aligned}
$$

where

$$
\begin{aligned}
& A_{n}=\frac{n !\left\{h_{n}(\alpha, \beta)\right\}^{\frac{1}{2}}}{\Gamma(\beta+n+1)}, \\
& B_{m}=\frac{m !\left\{h_{m}(\alpha,-\beta)\right\}^{\frac{1}{2}}}{\Gamma(-\beta+m+1)} .
\end{aligned}
$$

The series inversion formula then reads as follows.

If

$$
b_{m}^{*}=\frac{2^{\alpha+1}}{B_{m}} \frac{\sin \pi \beta}{\pi} \sum_{n=0}^{\infty} \frac{1}{(\alpha+m+n+1)(\beta-m+n)} \frac{a_{n}^{*}}{A_{n}},(\alpha>-1,0<\beta<1),
$$

then

$$
a_{n}^{*}=\frac{2^{\alpha+1}}{A_{n}} \frac{\sin \pi \beta}{\pi} \sum_{m=0} \frac{1}{(\alpha+m+n+1)(\beta-m+n)} \frac{b_{m}^{*}}{B_{m}},
$$

and vice versa. This result is valid if $\sum_{n=0}^{\infty}\left|a_{n}^{*}\right|^{2}<\infty$ or $\sum_{m=0}^{\infty}\left|b_{m}^{*}\right|^{2}<\infty$, each condition implying the other. Moreover

$$
\sum_{n=0}^{\infty}\left|a_{n}^{*}\right|^{2}=\sum_{m=0}^{\infty}\left|b_{m}^{*}\right|^{2}
$$

In the special case $\alpha=-\frac{1}{2}, \beta=\frac{1}{2}$, we have

$$
\begin{aligned}
A_{n} & =\frac{2}{2 n+1}(n=0,1,2, \cdots) \\
B_{0} & =1 \\
B_{m} & =\frac{1}{\sqrt{ } 2}(m=1,2, \cdots)
\end{aligned}
$$

and the inversion formulae reduce to 


$$
\begin{aligned}
& a_{n}^{*}=\frac{1}{\pi}\left\{\frac{\sqrt{2} b_{0}^{*}}{n+\frac{1}{2}}+\sum_{m=1}^{\infty}\left(\frac{1}{m+n+\frac{1}{2}}+\frac{1}{-m+n+\frac{1}{2}}\right) b_{m}^{*}\right\}, n=0,1,2, \cdots \\
& \sqrt{2} b_{0}^{*}=\frac{1}{\pi} \sum_{n=0}^{\infty} \frac{2}{n+\frac{1}{2}} a_{n}^{*} \\
& b_{m}^{*}=\frac{1}{\pi} \sum_{n=0}^{\infty}\left(\frac{1}{m+n+\frac{1}{2}}+\frac{1}{-m+n+\frac{1}{2}}\right) a_{n}^{*}, \quad m=1,2,3, \cdots
\end{aligned}
$$

Now if $a_{n}^{*}$ is defined for $n$ negative by the first of these formulae and $b_{m}^{*}$ for $m$ negative by the third, this gives

$$
\begin{aligned}
a_{-n-1}^{*} & =-a_{n}^{*}, & & n=0,1,2, \cdots \\
b_{-m}^{*} & =b_{m}, & & m=1,2, \cdots
\end{aligned}
$$

and the inversion formulae can be written as

$$
\begin{aligned}
a_{n}^{*} & =\frac{1}{\pi}\left\{\frac{\sqrt{2} b_{0}^{*}}{n+\frac{1}{2}}+\sum_{m=-\infty}^{\infty} \frac{b_{m}^{*}}{m+n+\frac{1}{2}}\right\}, n=0,1,2, \cdots \\
b_{m}^{*} & =\frac{1}{\pi} \sum_{n=-\infty}^{\infty} \frac{a_{n}^{*}}{m+n+\frac{1}{2}}, \quad m=1,2, \cdots \\
\sqrt{2} b_{0}^{*} & =\frac{1}{\pi} \sum_{n=-\infty}^{\infty} \frac{a_{n}^{*}}{n+\frac{1}{2}},
\end{aligned}
$$

which is a particular case of Titchmarsh's result [1], [2], if

$$
\alpha_{n}=\frac{\sin \pi \lambda}{\pi} \sum_{m=-\infty}^{\infty} \frac{\beta_{m}}{m+n+\lambda},
$$

then

$$
\beta_{m}=\frac{\sin \pi \lambda}{\pi} \sum_{n=-\infty}^{\infty} \frac{\alpha_{n}}{m+n+\lambda}
$$

The limiting case $\alpha \rightarrow \infty$ yields Linfoot and Shepherd's inversion formula. Using the familiar result that

we obtain

$$
\frac{\Gamma(\alpha+\mu)}{\Gamma(\alpha+v)} \sim \alpha^{\mu-v} \text { as } \alpha \rightarrow \infty,
$$

and finally

$$
\begin{aligned}
& A_{n} \sim\left\{\frac{2^{\alpha+\beta+1} \alpha^{-1-\beta} n !}{\Gamma(\beta+n+1)}\right\}^{\frac{1}{2}}, \\
& B_{m} \sim\left\{\frac{2^{\alpha-\beta+1} \alpha^{-1+\beta} m !}{\Gamma(-\beta+m+1)}\right\}^{\frac{1}{2}}
\end{aligned}
$$

$$
k_{m n} \rightarrow(-1)^{m+n} \frac{\sin \pi \beta}{\pi}\left\{\frac{\Gamma(-\beta+m+1)}{m !}\right\}^{\frac{1}{2}}\left\{\frac{\Gamma(\beta+n+1)}{n !}\right\}^{\frac{1}{2}} \frac{1}{\beta-m+n} .
$$


The resulting inversion formula is:

if

$b_{m}^{*}=\frac{\sin \pi \beta}{\pi}\left\{\frac{\Gamma(-\beta+m+1)}{m !}\right\}^{\frac{1}{2}} \sum_{n=0}^{\infty}\left\{\frac{\Gamma(\beta+n+1)}{n !}\right\}^{\frac{1}{2}} \frac{a_{n}^{*}}{\beta-m+n}, m=0,1,2, \cdots$,

then

$a_{n}^{*}=\frac{\sin \pi \beta}{\pi}\left\{\frac{\Gamma(\beta+n+1)}{n !}\right\}^{\frac{1}{2}} \sum_{m=0}^{\infty}\left\{\frac{\Gamma(-\beta+m+1)}{m !}\right\}^{\frac{1}{2}} \frac{b_{m}^{*}}{\beta-m+n}, m=0,1, \cdots$

and vice versa. This result is valid if $\sum_{n=0}^{\infty}\left|a_{n}^{*}\right|^{2}<\infty$ or $\sum_{m=0}^{\infty}\left|b_{m}^{*}\right|^{2}<\infty$, each condition implying the other. Moreover

$$
\sum_{n=0}^{\infty}\left|a_{n}^{*}\right|^{2}=\sum_{m=0}^{\infty}\left|b_{m}^{*}\right|^{2}
$$

(The fact that the limit of orthogonal transformations $K(\alpha)$ is also an orthogonal transformation is trivial.)

Linfoot and Shepherd [2] showed that if $\sum_{m=1}^{\infty}\left|\beta_{m}\right| \log m / m<\infty$, then the system of equations

$$
\beta_{m}=\frac{\sin \pi \lambda}{\pi} \sum_{n=0}^{\infty} \frac{\alpha_{n}}{n-m+\lambda}, \quad 0<\lambda<1,
$$

has as unique solution

$$
a_{n}=\frac{\sin \pi \lambda}{\pi} \frac{\Gamma(n+\lambda+1)}{n !} \sum_{n=0}^{\infty} \frac{\Gamma(-\lambda+m+1)}{m !} \frac{\beta_{m}}{n-m+\lambda} .
$$

Apart from the different convergence conditions this is equivalent to the result stated immediately above.

\section{References}

[1] E. C. Titchmarsh, 'Reciprocal formulae involving series and integrals', Math. Zeits. 25 (1926), 321-347.

[2] E. C. Titchmarsh, 'A series inversion formula', Proc. London Math. Soc. (2), 26 (1927), $1-11$.

[3] E. H. Linfoot and W. M. Shepherd, 'On a set of linear equations (II)', Quarterly J. Math. (Oxford) 10 (1939), 84-98.

[4] A. Erdelyi, W. Magnus, F. Oberhettinger, F. G. Tricomi, Tables of Integral Transforms, vol. 2 (McGraw-Hill, New York, 1954).

Mathematics Department

The University of New England

Armidale 